\title{
Innately robust yeast strains isolated from grape marc have a great potential for lignocellulosic ethanol production
}

\author{
Lorenzo Favaro • Marina Basaglia • Sergio Casella
}

Received: 2 December 2013 / Accepted: 23 January 2014 / Published online: 19 February 2014

(C) Springer-Verlag Berlin Heidelberg and the University of Milan 2014

\begin{abstract}
Bioethanol from lignocellulose is an attractive alternative to fossil fuels, and Saccharomyces cerevisiae is the most important ethanol producer. However, yeast cells are challenged by various environmental stresses during ethanol production on an industrial scale, and robust strains with a high tolerance to inhibitors, temperature and osmolality are needed for the effective feasibility of lignocellulosic ethanol. To search for such innately more resistant yeast, we selected grape marc as an extreme environment due to limited nutrients, exposure to solar radiation, temperature fluctuations, weak acids and ethanol. Using a temperature of $40^{\circ} \mathrm{C}$ as the key selection criterion, we isolated 120 novel S. cerevisiae strains from grape marc and found high ethanol yields (up to $92 \%$ of the theoretical maximum) when inoculated at $40^{\circ} \mathrm{C}$ in minimal media with a high sugar concentration. For the first time, this work assessed yeast tolerance to inhibitors at $40^{\circ} \mathrm{C}$, and the newly isolated yeast strains displayed interesting abilities to withstand increasing levels of single inhibitors or cocktails containing a mixture of inhibitory compounds. The newly isolated strains showed significantly higher fermentative abilities and tolerance to inhibitors than the industrial and commercial benchmark S. cerevisiae strains. The strong physiological robustness and fitness of a few of these $S$. cerevisiae yeast strains support their potential industrial application and encourage further studies in genetic engineering to enhance
\end{abstract}

L. Favaro $\cdot$ M. Basaglia $(\square) \cdot$ S. Casella

Department of Agronomy, Food Natural Resources, Animals and the

Environment (DAFNAE), University of Padova Agripolis, Viale dell'Università 16, 35020 Legnaro, PD, Italy

e-mail: marina.basaglia@unipd.it

L. Favaro

e-mail: lorenzo.favaro@unipd.it

S. Casella

e-mail: sergio.casella@unipd.it their ethanol performance in terms of rate and yield through the co-fermentation of all available carbon sources.

Keywords Lignocellulosic bioethanol - Yeast robustness · Thermo-tolerance $\cdot$ Inhibitor-tolerance $\cdot$ Grape marc $\cdot$ Strain selection

\section{Introduction}

Bioethanol production from renewable resources, such as lignocellulose, is considered a promising alternative to fossil fuels (Galbe and Zacchi 2002; Hamelinck et al. 2005). Ideally, the raw substrate for bioethanol production should be nonedible biomass, such as energy crops, spruce or birch, as well as agricultural by-products, including grain residues and sugarcane bagasse (Kim and Dale 2004; Demirbas 2009; Favaro et al. 2012a, 2013a).

Because native lignocellulosic biomass is highly refractory to degradation, pre-treatments are needed to make the cellulose more accessible to subsequent enzymatic saccharification. However, these methods also result in the co-production of a number of degradation compounds from the hemicellulose (mainly furfural and acetic acid), lignin (phenolic compounds) and cellulose [5-hydroxmethyl-2-furaldehyde (HMF)] fractions. These by-products, present in both the hydrolysate liquor and water-insoluble solid (WIS) fractions of the pre-treatment slurry, impair cellular metabolism and growth, thereby reducing the productivity of the process (Larsson et al. 1999; Almeida et al. 2007; den Haan et al. 2013); consequently, the development of robust yeast strains with improved production rates and resistance is of crucial importance.

A variety of detoxification strategies have been developed to remove or decrease the level of inhibitors from lignocellulosic hydrolysates. However, concerns regarding the techno- 
economic feasibility of these methods have been raised, namely, the additional expense of the process steps and the loss of fermentable sugars (Jönsson et al. 2013). Therefore, several countermeasures alternative to detoxification have been proposed to alleviate the challenges associated with inhibitors. Since the concentrations of toxic compounds and sugars in hydrolysates depend on the starting materials, as well as on the conditions during pre-treatment and hydrolysis (Galbe and Zacchi 2007), less recalcitrant feedstock can be selected and mild pre-treatment conditions can be applied (Galbe and Zacchi 2007; Favaro et al. 2013b). Alternatively, a number of avenues have been explored to make conditions more favourable for the fermenting microorganism (Wingren et al. 2003)

Advanced improvements in the optimisation of yeast robustness may require novel metabolic engineering tools, such as protein engineering, metabolic engineering and rational metabolic engineering, as elegantly described by Patnaik (2008) and Sagt (2013). Moreover, several breeding methods, including mutagenesis (Zhu et al. 2008), long-term adaptation (Balakumar et al. 2001; Liu et al. 2005), protoplast fusion, evolution engineering (Cakar 2005) and genome shuffling (Shi et al. 2009; Zhang and Geng 2012), have been previously employed to increase the ethanol production and thermotolerance of yeast. However, yeast strains exhibiting multitolerance to high temperature, inhibitors and ethanol levels have not yet been developed. Furthermore, laboratory strains were mainly utilised in most of these previous studies, and these may be difficult to use in industrial processes because of their generally low industrial fitness (Martin and Jönsson 2003; Garay-Arroyo et al. 2004).

One promising approach is to select for yeast strains with native resistance to inhibitors that could serve as a platform for engineering the ability to utilise xylose or arabinose as a carbon source for ethanol production. The use of naturally robust strains prevents interference with cloned genetic material, as could be the case when recombinant strains are subjected to hardening techniques.

In addition, there is a need to develop thermo-tolerant yeast capable of growth and fermentation at elevated temperatures compatible with optimal cellulase and hemicellulase activities to reduce the cost of cooling during fermentation. Thus, improving the robustness of Saccharomyces cerevisiae under the stress of heat and inhibitors is fundamental for the effective conversion of lignocellulose to ethanol.

Whereas many quality reports dealt with the pre-treatment of lignocellulosic materials tailored to maximise sugar release from the feedstock (reviewed in Galbe and Zacchi 2002, 2007), very few have considered yeast strains based on their innate resistance, fermentative traits and industrial scale adaptability (Lindén et al. 1992; Favaro et al. 2012a, 2013a). Moreover, the targets of previous screening or selection surveys for tolerant $S$. cerevisiae yeast were mainly individual stresses, such as high temperature (Babiker et al. 2010; Chen et al. 2013), weak acids or furans and aldehyde resistance (Martin and Jönsson 2003; Garay-Arroyo et al. 2004), while isolating and identifying yeast with a tolerance to multiple stresses apparently received little attention. However, employing naturally tolerant $S$. cerevisiae would, in fact, be a more realistic approach towards developing the second generation bioethanol industry, since it is the combined effect of the stressors that causes the greatest challenge to the success of industrial cellulosic ethanol production (van Maris et al. 2006).

To search for such robust, thermo-tolerant and strongly fermenting yeast, we assessed grape marc as an extreme environment because of the limited availability of nutrients, such as nitrogen and carbon, exposure to solar radiation, temperature fluctuations (between 20 and $45{ }^{\circ} \mathrm{C}$ ), low $\mathrm{pH}$ and concentrations of ethanol and weak acids (Favaro et al. 2013c). Using a temperature of $40{ }^{\circ} \mathrm{C}$ as a key selection criterion, we isolated $120 \mathrm{~S}$. cerevisiae strains from grape marc and evaluated these for their fermentative ability, as evidenced from glucose consumption and ethanol production in a minimal medium supplemented with high concentrations of glucose and xylose. The new yeast collection was subsequently screened for tolerance to inhibitors at $40{ }^{\circ} \mathrm{C}$ in yeast extract, peptone and dextrose (YPD) broth supplemented with increasing concentrations of single inhibitors or cocktails of inhibitory compounds. The effect of the culture $\mathrm{pH}$ on the tolerance of the yeast to inhibitor was also addressed.

\section{Materials and methods}

Yeast strains, isolation and genetic identification

The following four benchmark $S$. cerevisiae yeast strains were used in this study: the laboratory strain S. cerevisiae Y294, (ATCC 201160); the top fermenting beer strain S. cerevisiae DSM 70449; the commercial wine strain S. cerevisiae EC1118 obtained from Lallemand Fermented Beverages; the industrial strain $S$. cerevisiae 27P (Favaro et al. 2012b).

New yeast strains were isolated from grape marc collected from the 2011 vintage immediately following grape crushing, from a winery located in Italy. After storage for 30 days at the winery, $50 \mathrm{~g}$ of marc was dispersed in $500 \mathrm{~mL}$ of sterile physiological water $(0.85 \% \mathrm{NaCl})$ and the solution subjected to serial dilution before being plated on WL (Wallerstein Laboratory, Oxoid, UK) medium containing $200 \mu \mathrm{g} / \mathrm{mL}$ chloramphenicol (Sigma-Aldrich, USA) to contain bacterial growth and incubated at different temperatures (38, 40, 42 and $44{ }^{\circ} \mathrm{C}$ ) for $72 \mathrm{~h}$. Yeast colonies were isolated and then purified by growing on yeast and mould agar medium (YM; Oxoid) at $40{ }^{\circ} \mathrm{C}$ for $48 \mathrm{~h}$. Isolates were stored at $-80{ }^{\circ} \mathrm{C}$ in $\mathrm{YM}$ medium containing $20 \%(\mathrm{v} / \mathrm{v})$ glycerol. 
Genetic identification of the strains was achieved using a 750-bp fragment of the ITS1-5.8S-ITS2 sequence following Montrocher et al. (1998). A 5- $\mu \mathrm{L}$ aliquot of cell suspension was heated at $94{ }^{\circ} \mathrm{C}$ for $5 \mathrm{~min}$ and then subjected to 30 cycles of PCR amplification (initial denaturation at $94{ }^{\circ} \mathrm{C}$ for $30 \mathrm{~s}$, annealing at $56^{\circ} \mathrm{C}$ for $30 \mathrm{~s}$ and extension at $72{ }^{\circ} \mathrm{C}$ for $30 \mathrm{~s}$ ). Amplification products were checked by agarose gel electrophoresis and then subjected to Sanger sequencing using an ABI protocol for Taq-Dye Terminator Sequencing on an automated ABI377 sequencer (Applied Biosciences, USA). The obtained sequences were edited with Chromas Lite 2.1.1 (Technelysium Pty Ltd, Australia), and species identification was performed following BLASTn alignment (http://blast. ncbi.nlm.nih.gov/Blast) with those present in the GenBank public database. For species identification, a sequence similarity level of $98 \%$ was considered with a $>90 \%$ coverage.

Fermentative abilities of $S$. cerevisiae strains in minimal broth supplemented with high concentrations of sugars

In total, we evaluated $120 \mathrm{~S}$. cerevisiae strains for their fermentative ability in Must Nutritive Synthetic (MNS) minimal medium supplemented with different concentrations of glucose and/or xylose (20\% glucose, $10 \%$ glucose and $5 \%$ xylose) following the method described by Delfini (1995). MNS broth was specifically chosen because of its composition which can be considered to be quite similar to that of several poor industrial media (Dahod 1999) and to resemble the pre-industrial scale composition of bioethanol broth, where primarily $\left(\mathrm{NH}_{4}\right)_{2} \mathrm{SO}_{4}, \mathrm{MgSO}_{4} \cdot 7 \mathrm{H}_{2} \mathrm{O}$ and small amounts of yeast extract or corn steep liquor are usually added during the fermentation step (Graves et al. 2006; Sassner et al. 2008).

In brief, each glass serum bottle was filled with $100 \mathrm{~mL}$ of MNS medium and then sealed using rubber stoppers with a needle for the removal of $\mathrm{CO}_{2}$ produced during fermentation. Pre-cultures of $S$. cerevisiae strains, grown overnight in YPD broth, were collected, centrifuged and washed twice with sterile physiological water $(0.85 \% \mathrm{NaCl})$. Yeast cells were then inoculated, at an average cell concentration of $7.5 \times 10^{4}$ cells per millilitre, into serum bottles each containing $100 \mathrm{~mL}$ MNS broth. The incubation was performed in the static condition at 25 and $40{ }^{\circ} \mathrm{C}$. The $\mathrm{pH}$ of medium was set at 3.5 using $\mathrm{KOH}(5 \mathrm{M})$. Fermentative vigour was monitored daily by measuring bottle weight loss in relation to $\mathrm{CO}_{2}$ production. Results were reported, using a conversion factor of 2.118 (Delfini 1995), as grams of glucose utilised per liter of MNS. The experiments were carried out in triplicate. Samples were withdrawn after 7 and 21 days, filtered through a sieve (pore size $0.22 \mu \mathrm{m}$ ) and analyzed for their glucose and ethanol contents by high performance liquid chromatography
(HPLC) as indicated in the section Analytical methods and calculations.

Screening for tolerance to inhibitors

The newly isolated yeast strains together with the reference strains were evaluated for their tolerance to inhibitors YPD medium formulated with $20 \mathrm{~g} / \mathrm{L}$ glucose and increasing concentrations of weak acids (acetic, formic, lactic acid) and furans (furfural and HMF), supplemented as single compound or formulated as inhibitor cocktails.

The inhibitor levels were 1.80, 3.60, 5.40 and $7.20 \mathrm{~g} / \mathrm{L}$ for acetic acid (Merk, Germany); 0.61, 1.22, 1.83 and 2.44 g/L for formic acid (Sigma-Aldrich); 1.72, 3.45, 5.17 and $6.89 \mathrm{~g} / \mathrm{L}$ for lactic acid (Sigma-Aldrich); 0.69, 1.38, 2.08 and $2.77 \mathrm{~g} / \mathrm{L}$ for furfural (Sigma-Aldrich); 0.94, 1.86, 2.81 and $3.75 \mathrm{~g} / \mathrm{L}$ for HMF (Sigma-Aldrich). Inhibitors were also formulated into four cocktails (A, B, C and D) by mixing each increasing dose of each toxic compound, as reported in Table 1. Higher concentrations of a single inhibitor were also tested as follows: concentrations of 9.0, 10.8, 12.6 and $14.4 \mathrm{~g} / \mathrm{L}$ for acetic acid; $3.05,3.66 ; 4.27$ and $4.88 \mathrm{~g} / \mathrm{L}$ for formic acid; 3.46 and 4.15 $\mathrm{g} / \mathrm{L}$ for furfural; 4.69, 5.63 and $6.56 \mathrm{~g} / \mathrm{L}$ for HMF.

Yeast cells, grown overnight at $40{ }^{\circ} \mathrm{C}$ in YPD broth at $100 \mathrm{rpm}$, were incubated under aerobic conditions in $0.9 \mathrm{~mL}$ of medium at $40^{\circ} \mathrm{C}$. A low cell density $\left(7.5 \times 10^{5}\right.$ cells $\left./ \mathrm{mL}\right)$ was specifically used in this experiment to facilitate our search for robust yeast cells capable of withstanding both high temperature and high inhibitor(s) levels. The experiments were performed in triplicate.

After $40 \mathrm{~h}$, optical density at $600 \mathrm{~nm}\left(\mathrm{OD}_{600}\right)$ was measured. For each strain, the tolerance was evaluated as relative growth $\left(\mathrm{OD}_{600}\right.$ value, \%) by comparing the growth in the medium with and without the inhibitors.

The effects of $\mathrm{pH}$ on the tolerance of yeast to inhibitors were also considered. To this aim, the $\mathrm{pH}$ of YPD broth was left unchanged or, alternatively, adjusted to $4.5,5.0$ or 5.5, after the addition of the inhibitor(s), using $\mathrm{NaOH}(5 \mathrm{M})$ or $\mathrm{HCl}$ (5 M). This $\mathrm{pH}$ range was assessed because it is characteristic of many bioethanol production processes.

Table 1 Composition of synthetic inhibitor cocktails added to YPD broth

\begin{tabular}{lllll}
\hline Inhibitor $(\mathrm{g} / \mathrm{L})$ & Cocktail A & Cocktail B & Cocktail C & Cocktail D \\
\hline Acetic acid & 1.80 & 3.60 & 5.40 & 7.20 \\
Formic acid & 0.61 & 1.22 & 1.83 & 2.44 \\
Lactic acid & 1.72 & 3.45 & 5.17 & 6.89 \\
Furfural & 0.69 & 1.38 & 2.08 & 2.77 \\
HMF & 0.94 & 1.86 & 2.81 & 3.75 \\
\hline
\end{tabular}


Analytical methods and calculations

Samples were taken before and during MNS fermentation, filtered through a sieve (pore size $0.22 \mu \mathrm{m}$ ) and analysed by HPLC for glucose, xylose, xylitol, glycerol, acetic acid and ethanol. Monosaccharide analysis was performed with highperformance anion-exchange chromatography with pulsed amperometric detection. The system was equipped with a PA1 column and auto-sampler (Dionex Corporation, Sunnyvale, CA, USA) and the mobile phase used was $1 \mathrm{mM}$ $\mathrm{NaOH}$ at a flow rate of $1 \mathrm{~mL} / \mathrm{min}$ at room temperature (Favaro et al. 2013b).

Organic acids and ethanol were separated on an Aminex HPX-87H column (Bio-Rad, USA) at $65{ }^{\circ} \mathrm{C}$ with $5 \mathrm{mM}$ $\mathrm{H}_{2} \mathrm{SO}_{4}$ as the mobile phase, at a flow rate of $0.5 \mathrm{~mL} / \mathrm{min}$. The system (Shimadzu, Japan) was equipped with refractive index detector (Shimadzu) and a cation-H refill cartridge (BioRad).

All experiments were conducted in triplicate, and data were analyzed on Microsoft Excel using one-way analysis of variance. A probability value $(P)<0.05$ was considered to be statistically significant.

\section{Results and discussion}

Isolation and screening for efficient fermenting, thermoand osmo-tolerant $S$. cerevisiae strains

Although ability to produce ethanol by fermentation at high temperature is considered to be an essential phenotypic trait for a lignocellulosic bioethanol yeast (Banat et al. 1998; Chen et al. 2013), there is a limited number of reports on the screening and selection of yeast able to grow and ferment at or above $40^{\circ} \mathrm{C}$ (reviewed in Babiker et al. 2010). We therefore carried out yeast isolations on WL plates incubated at 38,40 , 42 and $44{ }^{\circ} \mathrm{C}$ to select for thermo-tolerant and robust yeast from grape marc, which is an unexplored source of microbial biodiversity. We found that a large number of colonies grew at $38^{\circ} \mathrm{C}$ and that there was limited or no growth at all at 42 and $44{ }^{\circ} \mathrm{C}$, respectively; therefore, we selected colonies from plates incubated at $40{ }^{\circ} \mathrm{C}$ for the isolation of thermo-tolerant strains for further study and genotyping. As described in the Materials and methods, 120 of these isolates showed similarities of the ITS1-5.8S-ITS2 sequence of more than $98 \%$ with the ITS1-5.8S-ITS2 sequence of $S$. cerevisiae type strains (Kurtzman and Robnett 2003) and were first screened for their ability to consume glucose at $40{ }^{\circ} \mathrm{C}$ in the MNS minimal broth supplemented with either $200 \mathrm{~g} / \mathrm{L}$ glucose or a combination of glucose $(100 \mathrm{~g} / \mathrm{L})$ and xylose $(50 \mathrm{~g} / \mathrm{L})$. These carbon sources were considered to be representative of the hexose and pentose content in most lignocellulosic hydrolysates (Galbe and Zacchi 2002; Hamelinck et al. 2005). In this study, the ability of the yeast to consume glucose was defined as fermenting vigour and expressed in terms of grams of glucose consumed per liter of MNS broth, as described in the Materials and methods.

Four benchmark strains of $S$. cerevisiae were included in this study. Two $S$. cerevisiae strains, namely DSM 70449 and 27P, had been previously adopted for ethanol production from different lignocellulosic substrates (Almeida et al. 2007; Favaro et al. 2012b), whereas the oenologically relevant S. cerevisiae strain EC1118 (Egli et al. 1998) and the laboratory strain Y294 (Martin and Jönsson 2003) were evaluated as additional benchmarks.

Our collection of newly isolated yeast strains produced interesting fermentative performances; Fig. 1 shows the fermenting ability of the 40 best performing yeast strains. At $25^{\circ} \mathrm{C}$ in MNS broth with $200 \mathrm{~g} / \mathrm{L}$ glucose, the majority of the selected isolates readily metabolised glucose (Fig. 1a). Of these, seven S. cerevisiae strains (F19, F47, F199, Fm84, Fm87, Fm88 and Fm92) exhibited outstanding fermentative performance, consuming all of the glucose. Surprisingly, the majority of the yeast strains isolated from grape marc showed better fermenting abilities than those of benchmark strains. In comparison, S. cerevisiae DSM 70449, the top fermenting beer yeast, showed a significantly lower sugar uptake, while the reference S. cerevisiae strains $27 \mathrm{P}$ and EC1118, although having the most prominent fermenting phenotype among the tested reference strains, metabolised glucose much slower. As expected, the laboratory S. cerevisiae Y294 exhibited only limited fermenting vigour, utilising no more than $110 \mathrm{~g} / \mathrm{L}$ glucose (Fig. 1a).

In the presence of xylose (Fig. 1b), only four S. cerevisiae strains (F66, F100, F199 and Fm92) were able to completely deplete glucose, while the other yeast strains did not use all of the glucose available in the broth, as confirmed also by HPLC analysis (data not shown). In general, the newly isolated strains, with the exception of $S$. cerevisiae Fm4, had better fermentative vigour than that the benchmark yeast.

As shown in Fig. 2, at higher temperature $\left(40^{\circ} \mathrm{C}\right)$ all of the strains showed a rapid consumption of glucose within the first days of culture, followed by a slowing down of fermentation, as detected by HPLC analysis; consequently, considerable amounts of glucose were still in the broth (data not shown). In MNS medium supplemented with $200 \mathrm{~g} / \mathrm{L}$ glucose, the control S. cerevisiae strains consumed up to $80 \mathrm{~g} / \mathrm{L}$ of glucose, while the majority of the new $S$. cerevisiae yeast strains had relatively higher fermenting abilities (Fig. 2a), with S. cerevisiae F55 and F156, capable of utilising $>120 \mathrm{~g} / \mathrm{L}$ glucose. The latter strain showed a fermenting vigour that was 1.5-fold higher than those of the benchmark yeast 27P and EC1118.

Similar behaviour was observed following the incubation of the yeast strains in MNS broth formulated with $100 \mathrm{~g} / \mathrm{L}$ glucose and $50 \mathrm{~g} / \mathrm{L}$ xylose, where only the reference 


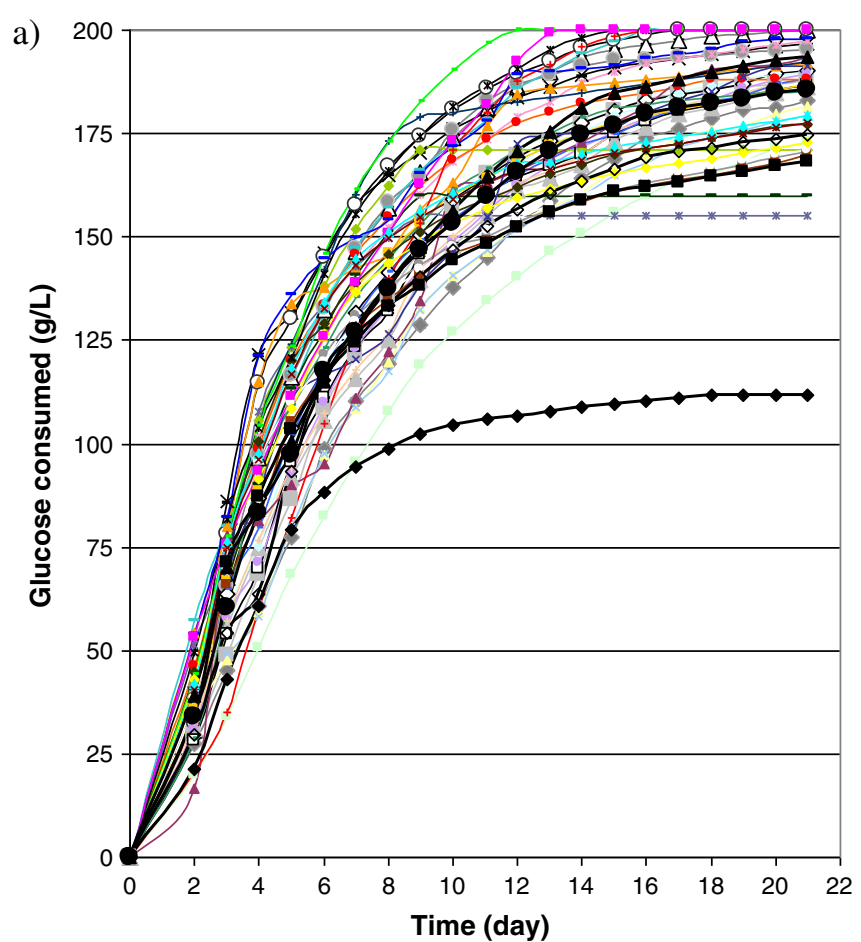

Fig. 1 Cumulative sugar utilisation (grams of glucose consumed per litre of MNS medium) of Saccharomyces cerevisiae strains following incubation at $25{ }^{\circ} \mathrm{C}$ in MNS medium with $200 \mathrm{~g} / \mathrm{L}$ glucose (a) and with $100 \mathrm{~g} / \mathrm{L}$

S. cerevisiae strain $27 \mathrm{P}$ showed fermentative vigour comparable to that measured for a cluster of the newly isolated

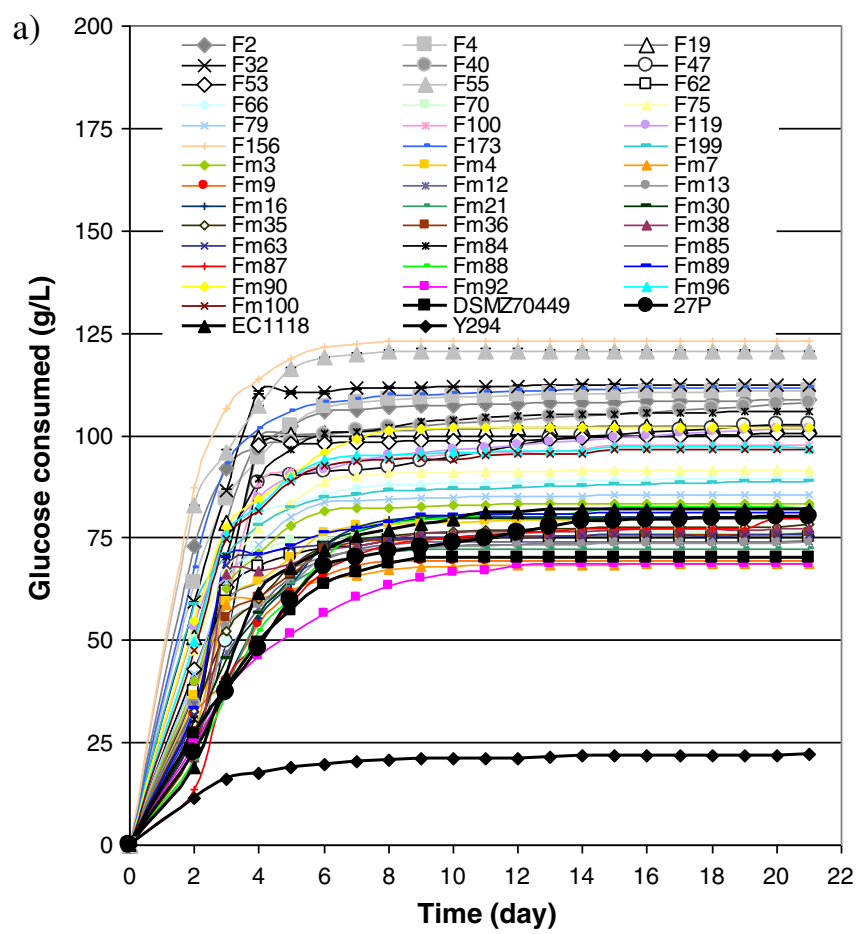

Fig. 2 Cumulative sugar utilisation (grams of glucose consumed per litre of MNS medium) of $S$. cerevisiae strains following incubation at $40{ }^{\circ} \mathrm{C}$ in MNS medium with $200 \mathrm{~g} / \mathrm{L}$ glucose (a) and with $100 \mathrm{~g} / \mathrm{L}$ glucose $+50 \mathrm{~g} /$

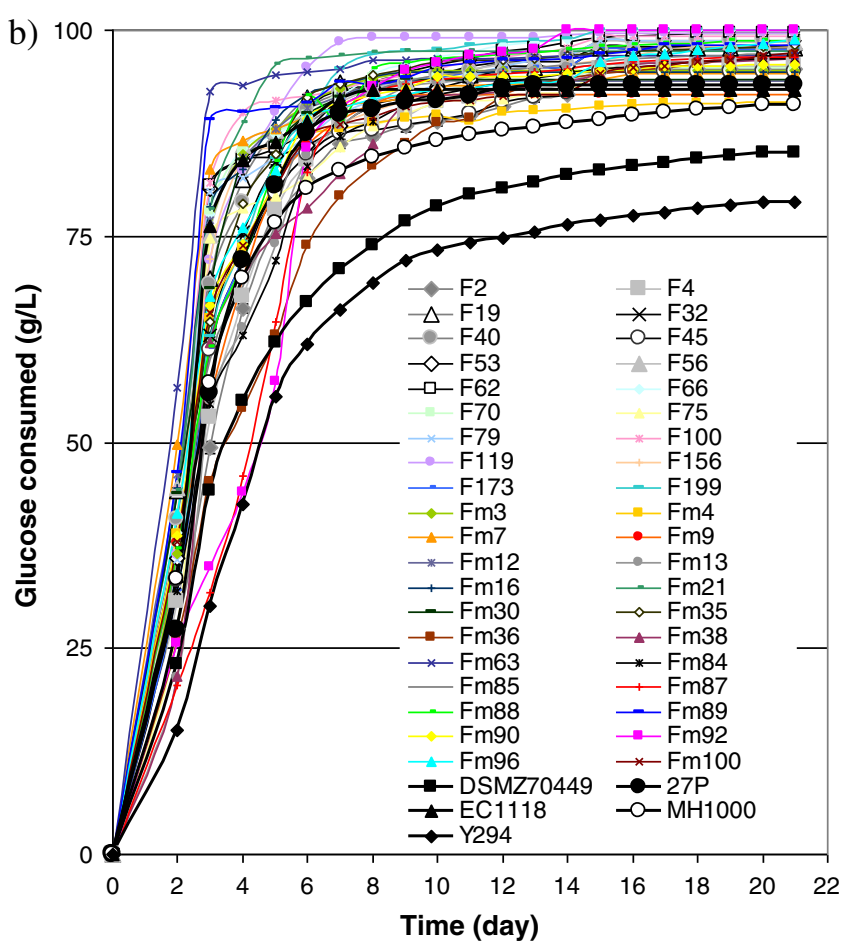

glucose $+50 \mathrm{~g} / \mathrm{L}$ xylose (b). All experiments were conducted in triplicate, and the relative standard error was always $<5 \%$ (not reported)

S. cerevisiae yeast strains (Fig. 2b). In contrast, the other reference yeast strains generally exhibited a low capability

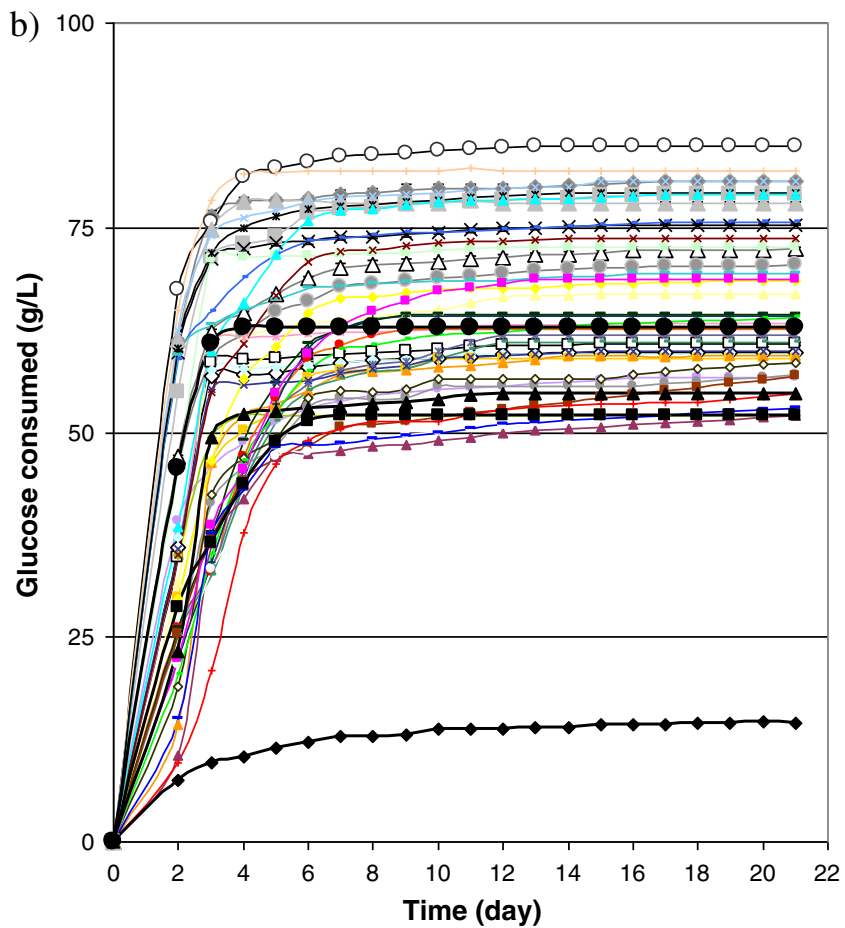

L xylose (b). All experiments were conducted in triplicate, and the relative standard error was always $<5 \%$ (not reported) 
of withstanding higher temperature and osmotic stress, since they consumed only up to $50 \mathrm{~g} / \mathrm{L}$ glucose while the results confirmed that laboratory strain Y294 also had the poorest fermenting ability at $40{ }^{\circ} \mathrm{C}$. Among the 40 selected new $S$. cerevisiae yeast strains assessed, the most proficient were S. cerevisiae F2, F47, F55, F79, F156 and Fm96, all of which showed a degree of glucose consumption that was much higher than those achieved by the reference yeast. For example, the glucose consumed by $S$. cerevisiae F47 was almost sixfold greater than that of the weakest control yeast strain, Y294, and 1.4-fold higher than that of the best reference yeast strain, 27P.

Ethanol performances at 25 and $40{ }^{\circ} \mathrm{C}$

Due to the large volume of data generated from the screening of the 120 newly isolated yeast strains, we performed a deeper analysis of the consumption of the carbon source and ethanol production only for the top eight strains in terms of fermentative vigour and for the three benchmark $S$. cerevisiae 27P, EC1118 and DSM 70449. The laboratory strain Y294, which exhibited poor fermentative vigour, was not included.

As reported in Table 2, when incubated at $25{ }^{\circ} \mathrm{C}$ in MNS medium containing $200 \mathrm{~g} / \mathrm{L}$ glucose, $S$. cerevisiae strains F47, Fm84 and Fm90 fermented glucose into ethanol with a peak yield of about $97 \%$ of the theoretical ( $0.51 \mathrm{~g}$ of ethanol per gram of glucose). This fermentative efficiency is higher than that recently reported for other $S$. cerevisiae strains cultured at high glucose concentrations (Ortiz-Muniz et al. 2010; He et al. 2012; Favaro et al. 2013b). Moreover, this value was significantly greater than that of the reference yeast DSM 70449, which yielded the lowest ethanol concentration (about $78 \mathrm{~g} /$ L), while the excellent suitability of both $S$. cerevisiae $27 \mathrm{P}$ and EC1118, producing $>90 \mathrm{~g} / \mathrm{L}$ ethanol, to be used in bioethanol and wine processes, respectively, was confirmed (Favaro et al. 2013d; Aceituno et al. 2012).

In the medium supplemented with $100 \mathrm{~g} / \mathrm{L}$ glucose and $50 \mathrm{~g} / \mathrm{L}$ xylose, the newly isolated strains achieved ethanol levels higher than those obtained by the reference strains. Interestingly, S. cerevisiae strains Fm84 and Fm90 produced about $48 \mathrm{~g} / \mathrm{L}$ ethanol, which was 1.09-fold higher than the alcohol concentration detected for the best control yeast, S. cerevisiae 27P. Comparing the ethanol yields obtained by the strains in both media (MNS with $200 \mathrm{~g} / \mathrm{L}$ glucose and MNS with $100 \mathrm{~g} / \mathrm{L}$ glucose $+50 \mathrm{~g} / \mathrm{L}$ xylose), it would appear that the presence of xylose in the MNS broth affected glucose uptake and ethanol production; indeed fermentation was slowed down in all of the yeast strains, but particularly in the reference strains, and all of the glucose available in the broth was not consumed (Table 2). For example, S. cerevisiae DSM 70449 had a much lower ethanol yield $(80 \%$ of the theoretical value) in MSN medium supplemented with both glucose and xylose than in MNS broth supplemented with only $200 \mathrm{~g} / \mathrm{L}$ glucose, thereby leaving a considerable amount

Table 2 Sugar consumption and product formation by selected Saccharomyces cerevisiae isolates and benchmark strains after 21 days of fermentation at $25^{\circ} \mathrm{C}$ in MNS broth supplemented with glucose alone $(200 \mathrm{~g} / \mathrm{L})$ or with glucose $(100 \mathrm{~g} / \mathrm{L})+$ xylose $(50 \mathrm{~g} / \mathrm{L})$

\begin{tabular}{|c|c|c|c|c|c|c|c|c|c|c|c|}
\hline \multirow[t]{2}{*}{ Medium } & \multicolumn{11}{|c|}{ Saccharomyces cerevisiae isolates and benchmark strains ${ }^{\mathrm{a}}$} \\
\hline & $\underline{27 \mathrm{P}}$ & $\underline{\mathrm{EC} 1118}$ & $\underline{\text { DSM } 70449}$ & $\mathrm{~F} 2$ & $\mathrm{~F} 4$ & F32 & F47 & F156 & F173 & Fm84 & Fm90 \\
\hline \multicolumn{12}{|c|}{ MNS with $20 \%$ glucose } \\
\hline Glucose $(\mathrm{g} / \mathrm{L})$ & 5.8 & 6.6 & 31.0 & 11.7 & 2.3 & 10.4 & - & 9.7 & 11.5 & - & 24.1 \\
\hline Glycerol (g/L) & 5.8 & 6.8 & 5.9 & 4.3 & 4.4 & 5.1 & 3.6 & 5.8 & 4.5 & 6.0 & 4.5 \\
\hline Ethanol (g/L) & 93.7 & 94.2 & 78.1 & 90.9 & 97.2 & 90.6 & 99.0 & 92.5 & 89.3 & 99.3 & 85.6 \\
\hline Ethanol yield ${ }^{\mathrm{b}}$ & 0.48 & 0.49 & 0.46 & 0.48 & 0.49 & 0.48 & 0.50 & 0.49 & 0.47 & 0.50 & 0.49 \\
\hline Ethanol yield $(\%)^{\mathrm{c}}$ & 94 & 96 & 91 & 95 & 96 & 94 & 97 & 96 & 93 & 97 & 97 \\
\hline \multicolumn{12}{|c|}{ MNS with $10 \%$ glucose and $5 \%$ xylose } \\
\hline Glucose $(\mathrm{g} / \mathrm{L})$ & 5.1 & 7 & 14.8 & 4.5 & 4.3 & 1.9 & 3.7 & 2.2 & 2.2 & - & 1.5 \\
\hline Xylose (g/L) & 48.2 & 45 & 46.7 & 48.7 & 47.1 & 45.2 & 44 & 47.1 & 48.3 & 48.1 & 46.5 \\
\hline Xylitol (g/L) & 1.7 & 4.8 & 2.9 & 1.3 & 2.6 & 4.6 & 5.8 & 2.4 & 1.6 & 1.4 & 3.6 \\
\hline Glycerol (g/L) & 3.6 & 3.9 & 3.6 & 3.2 & 3.3 & 3.7 & 3.2 & 3.2 & 2.9 & 2.8 & 3.4 \\
\hline Ethanol (g/L) & 43.8 & 43.4 & 34.9 & 44.2 & 45.4 & 46.5 & 44.5 & 47 & 45 & 48.2 & 47.5 \\
\hline Ethanol yield ${ }^{\mathrm{b}}$ & 0.46 & 0.47 & 0.41 & 0.46 & 0.47 & 0.47 & 0.46 & 0.48 & 0.46 & 0.48 & 0.47 \\
\hline Ethanol yield $(\%)^{\mathrm{c}}$ & 90 & 92 & 80 & 91 & 93 & 93 & 91 & 94 & 90 & 95 & 93 \\
\hline
\end{tabular}

All experiments were conducted in triplicate, and the relative standard error was always $<5 \%$ (not reported)

${ }^{a}$ Underlined strains are the control strains; all other strains (no underlining) are $S$. cerevisiae strains isolated from grape marc

${ }^{\mathrm{b}}$ Ethanol yield as gram ethanol/gram consumed glucose

${ }^{\mathrm{c}}$ Ethanol yield as $\%$ of theoretical maximum $(0.51 \mathrm{~g} / \mathrm{g}$ from glucose $)$ 
of glucose in the exhausted broth. However, further studies are in progress to investigate this interesting finding in more depth in order to elucidate the possible negative effect of high xylose concentrations on the glucose-to-ethanol conversion by S. cerevisiae. Nevertheless, as reported in Table 2, S. cerevisiae strains F156, Fm84 and Fm90 achieved remarkable ethanol yields (about $94 \%$ of the theoretical maximum) and their fermenting efficiencies are a valuable asset considering those displayed by the reference yeast and other $S$. cerevisiae strains (Matsushika et al. 2009; Zhang and Geng 2012).

When incubated at $40^{\circ} \mathrm{C}$ in MNS with $200 \mathrm{~g} / \mathrm{L}$ glucose, the selected strains produced ethanol concentrations ranging between 47.1 and $56.2 \mathrm{~g} / \mathrm{L}$, with the latter corresponding to an ethanol yield of $92 \%$ of the theoretical maximum. In comparison, the reference strains showed significantly lower fermenting efficiencies, with $S$. cerevisiae strain $27 \mathrm{P}$ being the most efficient at $86 \%$ of the theoretical maximum (Table 3).

In general, the benchmark yeast strains had consumed only half of the supplied glucose by the end of the fermentation period, pointing to ethanol inhibition, which is known to increase with temperature (Banat et al. 1998; Babiker et al. 2010). Similar results were observed in the MNS broth supplemented with $100 \mathrm{~g} / \mathrm{L}$ glucose and $50 \mathrm{~g} / \mathrm{L}$ xylose (Table 3). Although all of the yeast strains showed reduced ethanol efficiency in the presence of xylose, the production of ethanol by the newly isolated $S$. cerevisiae strains ranged from 32.4 to
$38.1 \mathrm{~g} / \mathrm{L}$, while the reference strains were characterised by a much lower fermenting performance. $S$. cerevisiae strains Fm84 and Fm90 exhibited the highest ethanol yields (about $92 \%$ of the theoretical value), which was 1.14 -fold higher than the yield achieved by the best control strain, 27P.

Overall, the fermentative parameters exhibited at $40{ }^{\circ} \mathrm{C}$ by the new collection of yeast isolated from grape marc are of great interest when compared to those reported in the literature (Babiker et al. 2010). Hacking et al. (1994) compared 55 yeast strains for glucose fermentation at elevated temperatures, achieving yields of $50 \%$ of the theoretical maximum with 12 strains at $40{ }^{\circ} \mathrm{C}$. Thermo-tolerant strains have also been isolated from hot climates or regions. Pellegrini and colleagues screened $457 \mathrm{~S}$. cerevisiae cultures, ultimately determining that the yeast DBVPG 1849, isolated from Ethiopian wine, was the most efficient fermenting strain at $40{ }^{\circ} \mathrm{C}$, with an ethanol yield of nearly $85 \%$ of the theoretical maximum (Pellegrini et al. 1999). Considering that the yeast DBVPG 1849 has the highest glucose-to-ethanol conversion yield at $40{ }^{\circ} \mathrm{C}$ described to date for a $S$. cerevisiae species, this is the first account of a $S$. cerevisiae yeast capable of fermenting glucose at $40{ }^{\circ} \mathrm{C}$ with ethanol yields close to $92 \%$ of the theoretical maximum in the presence of high concentrations of sugars. Such fermenting efficiency is even higher than that detected at $40{ }^{\circ} \mathrm{C}$ for UV mutants $(S$. cerevisiae UV1 and UV2) and few genome shuffled $S$. cerevisiae yeast evolved from the UV1 and UV2 strains (Shi et al. 2009).

Table 3 Sugar consumption and product formation by selected S. cerevisiae isolates and benchmark strains after 21 days of fermentation at $40{ }^{\circ} \mathrm{C}$ in MNS broth supplemented with glucose $(200 \mathrm{~g} / \mathrm{L})$ or with glucose $(100 \mathrm{~g} / \mathrm{L})+$ xylose $(50 \mathrm{~g} / \mathrm{L})$

\begin{tabular}{|c|c|c|c|c|c|c|c|c|c|c|c|}
\hline \multirow[t]{2}{*}{ Medium } & \multicolumn{11}{|c|}{ Saccharomyces cerevisiae isolates and benchmark strains ${ }^{\mathrm{a}}$} \\
\hline & $\underline{27 \mathrm{P}}$ & $\underline{\mathrm{EC} 1118}$ & $\underline{\text { DSM } 70449}$ & $\mathrm{~F} 2$ & F4 & F32 & F47 & F156 & F173 & Fm84 & Fm90 \\
\hline \multicolumn{12}{|c|}{ MNS with $20 \%$ glucose } \\
\hline Glucose $(\mathrm{g} / \mathrm{L})$ & 110.8 & 105.6 & 125.1 & 90.6 & 89.4 & 87.5 & 96 & 80.1 & 89.5 & 94 & 99.3 \\
\hline Glycerol (g/L) & 4.6 & 2.6 & 3.5 & 5 & 4.5 & 5.2 & 4 & 4.5 & 4.3 & 3.8 & 4 \\
\hline Ethanol (g/L) & 39 & 38.6 & 30 & 50.5 & 51.4 & 52.1 & 49.1 & 56.2 & 49.9 & 49.9 & 47.1 \\
\hline Ethanol yield ${ }^{\mathrm{b}}$ & 0.44 & 0.41 & 0.4 & 0.46 & 0.47 & 0.46 & 0.47 & 0.47 & 0.45 & 0.47 & 0.47 \\
\hline Ethanol yield $(\%)^{\mathrm{c}}$ & 86 & 80 & $78.00 \%$ & 91 & 91 & 91 & 92 & 92 & 89 & 92 & 92 \\
\hline \multicolumn{12}{|c|}{ MNS with $10 \%$ glucose and $5 \%$ xylose } \\
\hline Glucose (g/L) & 38.1 & 43.4 & 47.8 & 24.4 & 21 & 26.6 & 16.6 & 18.5 & 25.2 & 21 & 26 \\
\hline Xylose (g/L) & 47.4 & 46.7 & 46.3 & 48.5 & 46.8 & 46 & 46.9 & 48.3 & 49.3 & 49.1 & 48.5 \\
\hline Xylitol (g/L) & 2.2 & 2.1 & 2.8 & 3.3 & 3 & 3.8 & 3 & 3.8 & 3.6 & 2.5 & 2.5 \\
\hline Glycerol (g/L) & 3 & 2.5 & 2.8 & 3.2 & 2.7 & 3.2 & 3.3 & 2.7 & 3 & 2.5 & 2.9 \\
\hline Ethanol (g/L) & 25.5 & 22.7 & 19.5 & 33.9 & 34.3 & 32.4 & 38.1 & 38 & 34.1 & 37.3 & 34.6 \\
\hline Ethanol yield ${ }^{\mathrm{b}}$ & 0.41 & 0.4 & 0.37 & 0.45 & 0.45 & 0.44 & 0.46 & 0.47 & 0.45 & 0.47 & 0.47 \\
\hline Ethanol yield $(\%)^{\mathrm{c}}$ & 81 & 79 & 73 & 88 & 88 & 87 & 89 & 91 & 89 & 92 & 92 \\
\hline
\end{tabular}

All experiments were conducted in triplicate, and the relative standard error was always $<5 \%$ (not reported)

${ }^{a}$ Underlined strains are the control strains; all other strains (no underlining) are $S$. cerevisiae strains isolated from grape marc

${ }^{\mathrm{b}}$ Ethanol yield as gram ethanol/gram consumed glucose

${ }^{\mathrm{c}}$ Ethanol yield as \% of theoretical maximum $(0.51 \mathrm{~g} / \mathrm{g}$ from glucose) 
In previous studies, the thermo-tolerance of $S$. cerevisiae strains has been far screened by incubating the strains in complex media, such as yeast extract, peptone, glucose (YPD) and/or similarly composed broths (Hacking et al. 1994; Pellegrini et al. 1999; Shi et al. 2009; Babiker et al. 2010). Consequently, the fermenting abilities of the strains selected in our study are even more significant considering that (1) they were achieved in MNS minimal broth, whose composition is quite similar to that of several industrial broths (Dahod 1999) and 2) the fermentations were based on low initial inoculum size (about $10^{5}$ cells $/ \mathrm{mL}$ ).

Tolerance to inhibitors in YPD broth

The 120 newly isolated S. cerevisiae strains together with the four benchmark yeast strains were studied for their growth at $40{ }^{\circ} \mathrm{C}$ in the presence of increasing concentrations of inhibitory compounds (weak acids and furans), either formulated as a single toxic component or combined in inhibitor cocktails. Aerobic growth in the presence of inhibitors was chosen as the parameter to evaluate the yeast collection in terms of inhibitor tolerance.

The first set of experiments were conducted without adjusting the $\mathrm{pH}$ value after the addition of the inhibitor(s) and, consequently, the $\mathrm{pH}$ dropped mainly due to the addition of weak acids. This allowed us to screen the strains for the ability to withstand mostly the undissociated acidic forms. This experimental rationale turned out to be useful in selecting the most tolerant yeast as $S$. cerevisiae strains generally displayed strong but variable tolerance phenotypes to each inhibitory compound and, above all, to the inhibitor cocktails (data not shown). Among the aliphatic acids tested, lactic acid did not affect cell growth to any extent since in the presence of the highest amount of lactic acid considered in our study $(6.89 \mathrm{~g} /$ L), all of the strains produced relative growth values statistically similar to those obtained in the absence of the acid. Similar results have been recently reported by Albers and Larsson (2009) who found that lactic acid added to YPD in the range of 0 to $8 \mathrm{~g} / \mathrm{L}$ did not hinder the growth and metabolism of other laboratory and industrial S. cerevisiae strains. Therefore, additional experiments using this weak acid as the sole inhibitory compound are not reported here, although we continued to use lactic acid as a component of the inhibitor cocktails as it could act as additional acidic stress on yeast metabolism.

The addition of acetic and formic acid reduced yeast growth although the toxic effects of these acids were lower than those powered by furans. Interestingly, the hindering effects of the cocktails were powerful as the majority of the 124 strains screened were able to withstand only cocktail formulation $\mathrm{A}$, which contained the combination of the lowest amount of each inhibitor (data not shown).

To further investigate the tolerance to yeast strains to inhibitors, we evaluated ten S. cerevisiae strains, selected on the basis of their tolerance to each toxic compound, for their
Table 4 Influence of weak acids (acetic and formic acid) and furans (furfural and HMF) on growth at $40^{\circ} \mathrm{C}$ in YPD medium of selected newly isolated $S$. cerevisiae strains and the benchmark $S$. cerevisae 27P, EC1118, DMS70449 and Y294. After inhibitor(s) addition $\mathrm{pH}$ medium was adjusted to 4.5 . Values, reported as relative growth (\%) of the optical density measured for each strain after $40 \mathrm{~h}$ growth in YPD without inhibitor, are the means of three replicates. Standard error was always less than $4 \%$ (not reported). Bold and grey fonts are used for values equal to or higher than 90 and equal to or lower than 50 , respectively

\begin{tabular}{|c|c|c|c|c|c|c|c|c|c|c|c|c|c|c|c|c|}
\hline Inhibitor & $\mathrm{g} / \mathrm{L}$ & $\mathbf{m M}$ & $\underline{27 P}$ & $\underline{\text { EC1118 }}$ & DSM 70449 & $\underline{\mathbf{Y 2 9 4}}$ & F156 & Fm3 & Fm12 & Fm30 & Fm38 & Fm64 & Fm85 & Fm89 & Fm90 & Fm96 \\
\hline \multirow[t]{4}{*}{ Acetic acid } & 1.80 & 30 & 95 & 95 & 90 & 92 & 93 & 95 & 98 & 91 & 98 & 98 & 96 & 99 & 97 & 97 \\
\hline & 3.60 & 60 & 93 & 93 & 87 & 88 & 92 & 93 & 96 & 88 & 95 & 98 & 96 & 98 & 97 & 94 \\
\hline & 5.40 & 90 & 90 & 85 & 83 & 83 & 89 & 91 & 94 & 87 & 92 & 94 & 93 & 96 & 94 & 92 \\
\hline & 7.20 & 120 & 82 & 78 & 74 & 72 & 85 & 89 & 93 & 86 & 90 & 91 & 90 & 93 & 91 & 90 \\
\hline \multirow[t]{4}{*}{ Formic acid } & 0.61 & 13 & 99 & 99 & 96 & 96 & 99 & 100 & 100 & 100 & 100 & 100 & 100 & 100 & 100 & 100 \\
\hline & 1.22 & 27 & 99 & 99 & 94 & 96 & 98 & 99 & 100 & 100 & 100 & 100 & 100 & 100 & 100 & 99 \\
\hline & 1.83 & 40 & 98 & 99 & 94 & 96 & 98 & 99 & 100 & 100 & 99 & 99 & 100 & 100 & 100 & 98 \\
\hline & 2.44 & 53 & 98 & 99 & 93 & 95 & 97 & 98 & 100 & 98 & 98 & 98 & 100 & 100 & 100 & 98 \\
\hline \multirow[t]{4}{*}{ Furfural } & 0.69 & 7 & 95 & 85 & 84 & 89 & 88 & 85 & 90 & 85 & 94 & 97 & 86 & 93 & 90 & 90 \\
\hline & 1.38 & 14 & 83 & 76 & 64 & 83 & 80 & 82 & 88 & 83 & 92 & 95 & 82 & 90 & 87 & 88 \\
\hline & 2.08 & 22 & 70 & 22 & 44 & 28 & 70 & 77 & 87 & 56 & 91 & 91 & 81 & 89 & 86 & 68 \\
\hline & 2.77 & 29 & 47 & 8 & 18 & 0 & 51 & 63 & 25 & 20 & 43 & 57 & 78 & 85 & 85 & 52 \\
\hline \multirow[t]{4}{*}{$\overline{\mathrm{HMF}}$} & 0.94 & 7 & 95 & 89 & 86 & 85 & 90 & 90 & 93 & 91 & 95 & 85 & 91 & 87 & 89 & 92 \\
\hline & 1.86 & 15 & 88 & 81 & 79 & 78 & 81 & 87 & 91 & 83 & 92 & 71 & 81 & 75 & 78 & 87 \\
\hline & 2.81 & 22 & 75 & 65 & 66 & 60 & 75 & 82 & 87 & 67 & 87 & 69 & 69 & 73 & 75 & 74 \\
\hline & 3.75 & 30 & 51 & 45 & 44 & 35 & 55 & 70 & 76 & 45 & 82 & 40 & 61 & 70 & 72 & 56 \\
\hline \multirow[t]{4}{*}{ Cocktail } & A & & 86 & 75 & 75 & 81 & 78 & 76 & 87 & 83 & 88 & 84 & 77 & 90 & 88 & 83 \\
\hline & B & & 79 & 66 & 41 & 43 & 65 & 68 & 77 & 61 & 76 & 77 & 70 & 78 & 78 & 70 \\
\hline & $\mathrm{C}$ & & 50 & 20 & 16 & 17 & 51 & 66 & 69 & 28 & 62 & 72 & 64 & 70 & 65 & 60 \\
\hline & $\mathrm{D}$ & & 9 & 0 & 0 & 0 & 0 & 8 & 7 & 4 & 8 & 18 & 11 & 16 & 14 & 5 \\
\hline
\end{tabular}

Underlined strains are the control strains; all other strains (no underlining) are S. cerevisiae strains isolated from grape marc 
ability to grow at $40^{\circ} \mathrm{C}$ in YPD broth with the $\mathrm{pH}$ adjusted to 4.5 after addition of the inhibitors. Reference yeast were included in the experiment for comparison (Table 4).

On a molar basis, formic acid and acetic acid had a similar inhibiting effect on cell growth. Accordingly, when exposed to the highest dose of formic acid $(53 \mathrm{mM})$, the yeast strains showed relative growth values ranging from 93 to $100 \%$, similar to those detected in the broth supplemented with $60 \mathrm{mM}$ acetic acid. However, additional doses of acetic acid impaired cell growth and, when exposed to the highest concentration $(120 \mathrm{mM})$, the selected yeast strains showed relative growth values ranging from 85 to $93 \%$ of the growth achieved in the medium without acetic acid, with strains Fm12 and Fm89 showing the most promise. The performance of the control strains exhibited a similar trend, although their values were in most cases lower than those exhibited by the newly isolated yeast strains. Among the reference yeast, the laboratory strain S. cerevisiae Y294 had a tolerance to the inhibitors that was comparable with that of the wildtype yeasts S. cerevisiae EC1118 and DSM 70449. This result is in accordance with those reported on laboratory strains

Table 5 Influence of weak acids (acetic and formic acid) and furans (furfural and HMF) on growth at $40{ }^{\circ} \mathrm{C}$ in YPD medium of selected S. cerevisiae strains and of the most tolerant control yeast 27P. After inhibitor(s) addition $\mathrm{pH}$ medium was adjusted to 4.5 or to 5.0 and 5.5 in the case of the cocktails A, B, C, D. Values, reported as relative growth exhibiting few phenotypic traits comparable to those of robust industrial yeast (Martin and Jönsson 2003).

Of the furans tested, furfural was the most toxic, as evident from the $30 \%$ decrease in relative growth of the yeast strains that was observed following the addition of $2.08 \mathrm{~g} / \mathrm{L}$ furfural. Strains Fm89 and Fm90 exhibited the greatest degree of tolerance at $2.77 \mathrm{~g} / \mathrm{L}$ furfural. Similarly, supplementation with HMF also resulted in severe decreases in growth, even though these responses were not as striking as those with furfural. As a result, in the presence of $2.81 \mathrm{~g} / \mathrm{L} \mathrm{HMF}$, the yeast strains showed relative growth values ranging from 67 to $87 \%$ of the culture growth achieved in the medium without this inhibitor, with the strains Fm12 and Fm38 showing the highest level of tolerance also at $3.75 \mathrm{~g} / \mathrm{L}$.

Inhibitor cocktails, formulated as described in Table 1, severely hindered cell growth, with the benchmark yeast strains being the most sensitive (Table 4). Although cocktails $\mathrm{A}$ and $\mathrm{B}$ generally resulted in strong growth inhibition, cocktails $\mathrm{C}$ and $\mathrm{D}$ had the highest negative effects on yeast growth. Nevertheless, S. cerevisiae strains Fm12, Fm64 and Fm89, when exposed to cocktail $\mathrm{C}$, exhibited the highest degree of

(\%) of the optical density measured for each strain after $40 \mathrm{~h}$ growth in YPD without inhibitor, are the means of three replicates. Standard error was always less than $4 \%$ (not reported). Bold and grey fonts are used for values equal to or higher than 90 and equal to or lower than 50 , respectively.

\begin{tabular}{lcccccccc}
\hline Inhibitor & $\mathbf{g} / \mathbf{L}$ & $\mathbf{m M}$ & $\underline{\mathbf{2 7 P}}$ & $\mathbf{F m 1 2}$ & $\mathbf{F m 3 8}$ & $\mathbf{F m 6 4}$ & $\mathbf{F m 8 9}$ & $\mathbf{F m 9 0}$ \\
\hline Acetic acid & 9.00 & 150 & 69 & 80 & 78 & 76 & 78 & 77 \\
& 10.80 & 180 & 21 & 68 & 58 & 68 & 63 & 63 \\
& 12.60 & 210 & 5 & 56 & 23 & 55 & 50 & 53 \\
\hline Formic acid & 14.40 & 240 & 0 & 10 & 0 & 6 & 5 & 3 \\
\hline Furfural & 3.05 & 66 & $\mathbf{9 2}$ & $\mathbf{9 5}$ & 85 & $\mathbf{9 6}$ & $\mathbf{9 6}$ & $\mathbf{9 5}$ \\
& 3.66 & 79 & $\mathbf{9 1}$ & $\mathbf{9 3}$ & 80 & $\mathbf{9 4}$ & $\mathbf{9 7}$ & $\mathbf{9 3}$ \\
& 4.27 & 92 & 82 & 86 & 76 & $\mathbf{9 3}$ & $\mathbf{9 3}$ & $\mathbf{9 0}$ \\
& 4.88 & 105 & 70 & 88 & 72 & 86 & 88 & 87 \\
\hline HMF & 3.46 & 36 & 40 & 0 & 0 & 76 & 0 & 0 \\
& 4.15 & 43 & 0 & 0 & 0 & 0 & 0 & 0 \\
\hline Cocktail pH 5.0 & 4.69 & 38 & 15 & 58 & 58 & 30 & 55 & 56 \\
& 5.63 & 46 & 0 & 21 & 25 & 14 & 18 & 29 \\
& A.56 & 54 & 0 & 5 & 8 & 0 & 0 & 0 \\
\hline A & & 89 & $\mathbf{9 1}$ & $\mathbf{9 2}$ & $\mathbf{9 2}$ & $\mathbf{9 3}$ & $\mathbf{9 2}$ \\
& B & & 84 & 84 & 82 & 83 & 83 & 81 \\
& C & & 71 & 74 & 70 & 76 & 75 & 78 \\
& D & & 36 & 59 & 62 & 60 & 63 & 64 \\
\hline & A & & $\mathbf{9 4}$ & $\mathbf{9 6}$ & $\mathbf{9 6}$ & $\mathbf{9 5}$ & $\mathbf{9 4}$ & $\mathbf{9 6}$ \\
& B & & 87 & 88 & 88 & 87 & 86 & 88 \\
& D & & 75 & 80 & 78 & 81 & 78 & 79 \\
& & 51 & 73 & 70 & 70 & 72 & 74 \\
\hline
\end{tabular}

Underlined strain is the control strain; all other strains (no underlining) are S. cerevisiae strains isolated from grape marc 
tolerance, with a relative growth of about $70 \%$. In contrast, cocktail $\mathrm{D}$, formulated with $7.20 \mathrm{~g} / \mathrm{L}$ acetic acid, $2.44 \mathrm{~g} / \mathrm{L}$ formic acid, $6.89 \mathrm{~g} / \mathrm{L}$ lactic acid, $2.77 \mathrm{~g} / \mathrm{L}$ furfural and 3.75 $\mathrm{g} / \mathrm{L}$ HMF, supported only a slight growth by a few of the tested strains, suggesting that each inhibitory compound may have synergistically challenged the yeast to grow under this multiple environmental stress.

To investigate the tolerance to inhibitors of the five most promising S. cerevisiae strains, we supplemented YPD broth with higher concentrations of each toxic compound. We also evaluated the tolerance of the strains to inhibitors in cocktails $\mathrm{A}, \mathrm{B}, \mathrm{C}$ and $\mathrm{D}$, adjusting the $\mathrm{pH}$ to 5.0 and 5.5. The reference yeast strain $27 \mathrm{P}$, which had a tolerance to inhibitors that was generally comparable to that of the newly isolated strains, was included in the experiment (Table 5).

The yeast strains had high relative growth values at the tested acetic acid levels $(9,10.8,12.6$ and $14.4 \mathrm{~g} / \mathrm{L})$. This finding can be explained in the context of the specific environment they originated from. Grape marc is quite rich in acetic acid (Ribereau-Gayon et al. 2007; Favaro et al. 2013c). In contrast, as reported in Table 5, the reference S. cerevisiae strain $27 \mathrm{P}$ was revealed to be the most sensitive of the tested yeast strains.

Strikingly, the newly isolated strains were able to grow in the presence of higher formic concentrations (Table 5) with relative growth values ranging from 72 to $88 \%$ with $4.88 \mathrm{~g} / \mathrm{L}$ formic acid. The most resistant strains, Fm64, Fm89 and Fm90, also tolerated further increases in the concentration of this aliphatic acid of up to $8.0 \mathrm{~g} / \mathrm{L}$ (data not shown).

Only strains $S$. cerevisiae 27P and Fm64 grew in YPD supplemented with $3.46 \mathrm{~g} / \mathrm{L}$ furfural, with these newly isolated strains showing significantly higher resistance than the reference yeast. HMF was less toxic than furfural as, with the exception of the industrial strain 27P, the yeast had the potential to grow in the presence of up to $5.63 \mathrm{~g} / \mathrm{L} \mathrm{HMF}$ (Table 5).

The results obtained with four inhibitor cocktails at $\mathrm{pH} 5.0$ and 5.5 show that for each strain the higher $\mathrm{pH}$, the more pronounced the tolerance, with S. cerevisiae Fm89 and Fm90 exhibiting relative growth values of more than 63 and $72 \%$ at pH 5.0 and 5.5, respectively, in cocktail D. The reference yeast strain 27P was inhibited to a higher extent under identical conditions, showing, values of only 36 and $51 \%$, respectively.

Taking the results obtained with the cocktails at $\mathrm{pH} 4.5$ into consideration (Table 4), it clearly appears that $\mathrm{pH}$ plays a central role in the tolerance of yeast strains to inhibitors. As a result, the $\mathrm{pH}$ value(s) usually set in many biomass-toethanol processes on the basis of the optimal values for the commercial hydrolytic enzymes (amylases, cellulases, xylanases) should be carefully defined to also take into account the amount and type of inhibitors present in the starting or pre-treated materials in order to boost the tolerance of the yeast strain to the toxicity of the inhibitors.
Overall, the promising inhibitor tolerance phenotypes detected in YPD at different $\mathrm{pH}$ values were notable, as many published studies on $S$. cerevisiae inhibitor endurance used predominantly YPD or YPD-based media, but with the $\mathrm{pH}$ adjusted to higher values (up to 6.5), which would have decreased the inhibiting power of the aliphatic acids to which the cultures were exposed (Albers and Larsson 2009; Koppram et al. 2012). In addition, the reported surveys were conducted at $30^{\circ} \mathrm{C}$ while in our study the tolerance of yeast strains to inhibitors was evaluated for the first time at $40{ }^{\circ} \mathrm{C}$. Moreover, to facilitate our search for robust yeast strains, we specifically designed our study to start with a cell inoculum size (about $10^{6}$ cells $/ \mathrm{mL}$ ) tenfold lower than that normally used in similar experimental activities (Larsson et al. 2001; Martin and Jönsson 2003; Garay-Arroyo et al. 2004).

To our knowledge, this is the first account describing the isolation, characterisation and selection of $S$. cerevisiae yeast strains based on high fermenting abilities and inhibitor tolerance at $40{ }^{\circ} \mathrm{C}$. The new thermo-tolerant yeast collection screened for tolerance to inhibitors at $40{ }^{\circ} \mathrm{C}$ show a great potential to tolerate inhibitor(s) concentrations much higher than those exhibited by commercial and industrial yeast generally used in the biomass-to-ethanol route. For example, $S$. cerevisiae strain Fm90, selected as one of the best fermenting isolates (Tables 2 and 3), showed a promising inhibitor tolerance phenotype (Tables 4, 5). As a result, we have demonstrated that the choice of grape marc as a source of microbial diversity was effective in terms of isolating new strains capable of coping with the most significant stresses prevalent in large-scale bioethanol production. Moreover, the phenotypic differences observed between the screened yeast isolates indicated that the selection of strain is decisive when contemplating the design of a process involving fermentation in the presence of lignocellulosic hydrolysates.

The strong physiological robustness and fitness of a few of our newly isolated $S$. cerevisiae strains support their potential industrial application and encourage further genetic engineering to enhance their ethanol performance through the cofermentation of all available carbon sources.

Acknowledgments This work was supported by the research project entitled "Engineering Consolidated Bioprocessing yeast for the one-step conversion of cellulosic substrates into bioethanol" (University of Padova, Padova, Italy). Dr. Favaro is recipient of "Assegno di ricerca Senior" grant from the University of Padova. Stefania Zannoni (University of Padova) is acknowledged for the HPLC analysis.

\section{References}

Aceituno FF, Orellana M, Torres J, Mendoza S, Slater AW, Melo F, Agosin E (2012) Oxygen response of the wine yeast Saccharomyces cerevisiae EC1118 grown under carbon-sufficient, 
nitrogen-limited enological conditions. Appl Environ Microbiol 78: $8340-8352$

Albers E, Larsson C (2009) A comparison of stress tolerance in YPD and industrial lignocellulose-based medium among industrial and laboratory yeast strains. J Ind Microbiol Biotechnol 36:1085-1091

Almeida JRM, Modig T, Petersson A, Hahn-Hägerdal B, Liden G, Gorwa-Grauslund MF (2007) Increased tolerance and conversion of inhibitors in lignocellulosic hydrolysates by Saccharomyces cerevisiae. J Chem Technol Biotechnol 82:340-349

Babiker MAA, Hoshida H, Ano A, Nonklang S, Akada R (2010) Hightemperature fermentation: how can processes for ethanol production at high temperatures become superior to the traditional process using mesophilic yeast? Appl Microbiol Biotechnol 85:861-867

Balakumar S, Arasaratnam V, Balasubramaniam K (2001) Isolation and improvement of a thermotolerant Saccharomyces cerevisiae strain. World J Microbiol Biotechnol 17:739-746

Banat IM, Nigam P, Singh D, Marchant R, McHale AP (1998) Ethanol production at elevated temperatures and alcohol concentrations: Part I-Yeasts in general. World J Microbiol Biotechnol 14:809-821

Cakar ZP (2005) Evolutionary engineering of multiple-stress resistant Saccharomyces cerevisiae. FEMS Yeast Res 5:569-578

Chen Q, Fang Y, Zhao H, Zhang G, Jin Y (2013) Transcriptional analysis of Saccharomyces cerevisiae during high-temperature fermentation. Ann Microbiol 63:1433-1440

Dahod SK (1999) Raw material selection and medium development for industrial fermentation processes. In: Demain AL, Davies JE (eds) Manual of industrial microbiology and biotechnology, 2nd edn. ASM Press, Washington DC

Delfini C (1995) Scienza e tecnica di microbiologia enologica. Edizioni Il lievito, Asti

Demirbas MF (2009) Biorefineries for biofuel upgrading: a critical review. Appl Energy 86:S151-S161

den Haan R, Kroukamp H, Mert M, Bloom M, Görgens JF, van Zyl WH (2013) Engineering Saccharomyces cerevisiae for next generation ethanol production. J Chem Technol Biotechnol 88:983-991

Egli CM, Edinger WD, Mitrakul CM, Henich-Kling T (1998) Dynamics of indigenous and inoculated yeast populations and their effect on the sensory character of Riesling and Chardonnay wines. J Appl Microbiol 85:779-789

Favaro L, Basaglia M, Casella S (2012a) Processing wheat bran into ethanol using mild treatments and highly fermentative yeasts. Biomass Bioenergy 46:605-617

Favaro L, Jooste T, Basaglia M, Rose SH, Saayman M, Görgens JF, Casella S, van Zyl WH (2012b) Codon-optimized glucoamylase sGAI of Aspergillus awamori improves starch utilization in an industrial yeast. Appl Microbiol Biotechnol 95:957-968

Favaro L, Basaglia M, Trento A, van Rensburg E, García-Aparicio M, van Zyl WH, Casella S (2013a) Exploring grape marc as trove for new thermotolerant and inhibitor-tolerant Saccharomyces cerevisiae strains for second-generation bioethanol production. Biotechnol Biofuels 6:168

Favaro L, Basaglia M, van Zyl WH, Casella S (2013b) Using an efficient fermenting yeast enhances ethanol production from unfiltered wheat bran hydrolysates. Appl Energy 102:170-178

Favaro L, Corich V, Giacomini A, Basaglia M, Casella S (2013c) Grape marcs as unexplored source of new yeasts for future biotechnological applications. World J Microbiol Biotechnol 29:1551-1562

Favaro L, Jooste T, Basaglia M, Rose SH, Saayman M, Görgens JF, Casella S, van Zyl WH (2013d) Designing industrial yeasts for the consolidated bioprocessing of starchy biomass to ethanol. Bioengineered 4:1-6

Galbe M, Zacchi G (2002) A review of the production of ethanol from softwood. Appl Microbiol Biotechnol 59:618-628

Galbe M, Zacchi G (2007) Pre-treatment of lignocellulosic materials for efficient bioethanol production. Adv Biochem Eng Biotechnol 108: $41-65$
Garay-Arroyo A, Covarrubias AA, Clark I, Niño I, Gosset G, Martinez A (2004) Response to different environmental stress conditions of industrial and laboratory Saccharomyces cerevisiae strains. Appl Microbiol Biotechnol 63:734-741

Graves T, Narendranath NV, Dawson K, Power R (2006) Effect of pH and lactic or acetic acid on ethanol productivity by Saccharomyces cerevisiae in corn mash. J Ind Microbiol Biotechnol 33:469-474

Hacking AJ, Taylor IWF, Hanas CM (1994) Selection of yeast able to produce ethanol from glucose at $40^{\circ} \mathrm{C}$. Appl Microbiol Biotechnol 19:361-363

Hamelinck CN, Van Hooijdonk G, Faaij APC (2005) Ethanol from lignocellulosic biomass: techno-economic performance in short-, middle- and long-term. Biomass Bioenergy 28:384-410

He L-Y, Zhao X-Q, Bai F-W (2012) Engineering industrial Saccharomyces cerevisiae strain with the FLO1-derivative gene isolated from the flocculating yeast SPSC01 for constitutive flocculation and fuel ethanol production. Appl Energy 100:33-40

Jönsson LJ, Alriksson B, Nilvebrant NO (2013) Bioconversion of lignocellulose: inhibitors and detoxification. Biotechnol Biofuels 6:16

Kim S, Dale BE (2004) Global potential bioethanol production from wasted crops and crop residues. Biomass Bioenergy 26:361-375

Koppram R, Albers E, Olsson L (2012) Evolutionary engineering strategies to enhance tolerance of xylose utilizing recombinant yeast to inhibitors derived from spruce biomass. Biotechnol Biofuels 5:32

Kurtzman CP, Robnett CJ (2003) Phylogenetic relationships among yeasts of the 'Saccharomyces complex' determined from multigene sequence analyses. FEMS Yeast Res 3:417-432

Larsson S, Palmqvist E, Hahn-Hägerdal B, Tengborg C, Stenberg K, Zacchi G, Nilvebrant NO (1999) The generation of fermentation inhibitors during dilute acid hydrolysis of softwood. Enzyme Microb Technol 24:151-159

Larsson S, Cassland P, Jönsson LJ (2001) Development of a Saccharomyces cerevisiae strain with enhanced resistance to phenolic fermentation inhibitors in lignocellulose hydrolysates by heterologous expression of laccase. Appl Environ Microbiol 67:1163-1170

Lindén T, Peetre J, Hahn-Hägerdal B (1992) Isolation and characterization of acetic acid-tolerant galactose-fermenting strains of Saccharomyces cerevisiae from a spent sulfite liquor fermentation plant. Appl Environ Microbiol 58:1661-1669

Liu ZL, Slininger PJ, Gorsich SW (2005) Enhanced biotransformation of furfural and hydroxymethylfurfural by newly developed ethanologenic yeast strains. Appl Biochem Biotechnol 121:451-460

Martin C, Jönsson LJ (2003) Comparison of the resistance of industrial and laboratory strains of Saccharomyces and Zygosaccharomyces to lignocellulose-derived fermentation inhibitors. Enzyme Microb Technol 32:386-395

Matsushika A, Inoue H, Kodaki T, Sawayama S (2009) Ethanol production from xylose in engineered Saccharomyces cerevisiae strains: current state and perspectives. Appl Microbiol Biotechnol 84:37-53

Montrocher R, Verner MC, Briolay J, Gautier C, Marmeisse R (1998) Phylogenetic analysis of the Saccharomyces cerevisiae group based on polymorphisms of rDNA spacer sequences. Int J Syst Bacteriol 48:295-303

Ortiz-Muniz B, Carvajal-Zarrabal O, Torrestiana-Sanchez B, AguilarUscanga MG (2010) Kinetic study on ethanol production using Saccharomyces cerevisiae ITV-01 yeast isolated from sugarcane molasses. J Chem Technol Biotechnol 85:1361-1367

Patnaik R (2008) Engineering complex phenotypes in industrial strains. Biotechnol Prog 24:38-47

Pellegrini L, Cardinali G, Martini A (1999) Selection of Saccharomyces cerevisiae strains able to ferment at supraoptimal temperatures. Ann Microbiol 49:55-65

Ribereau-Gayon P, Dubourdieu D, Doneche B, Lonvaud A (2007) Traite d'oenologie I. Dunod, Paris

Sagt CMJ (2013) Systems metabolic engineering in an industrial setting. Appl Microbiol Biotechnol 97:2319-2326 
Sassner P, Galbe M, Zacchi G (2008) Techno-economic evaluation of bioethanol production from three different lignocellulosic materials. Biomass Bioenergy 32:422-430

Shi D-J, Wang C-L, Wang K-M (2009) Genome shuffling to improve thermotolerance, ethanol tolerance and ethanol productivity of Saccharomyces cerevisiae. J Ind Microbiol Biotechnol 36:139-147 van Maris AJ, Abbott DA, Bellissimi E, van den Brink J, Kuyper M, Luttik MA, Wisselink HW, Scheffers WA, van Dijken JP, Pronk JT (2006) Alcoholic fermentation of carbon sources in biomass hydrolysates by Saccharomyces cerevisiae: current status. Antonie Van Leeuwenhoek 90:391-418
Wingren A, Galbe M, Zacchi G (2003) Techno-economic evaluation of producing ethanol from softwood: comparison of SSF and SHF and identification of bottlenecks. Biotechnol Prog 19: $1109-1117$

Zhang W, Geng A (2012) Improved ethanol production by a xylosefermenting recombinant yeast strain constructed through a modified genome shuffling method. Biotechnol Biofuels 5:46

Zhu H, Xu JZ, Shi J, Zhang R, Sun XY, Zhu RR, Yao SD, Wang SL (2008) Mutation breeding for productive yeast strains through a novel method: High-energy-pulse-electron-beam. Ann Microbiol 58:549-553 